\title{
Numerical Solution of the Kirchhoff Plate Bending Problem with BEM
}

\author{
V. V. Zozulya \\ Departamento de Materiales Compuestos, Centro de Investigación Científica de Yucatán A.C., Calle 43, No. 130, \\ Colonia Chuburná de Hidalgo, 97200 Mérida, YC, Mexico \\ Correspondence should be addressed to V. V.Zozulya, zozulya@cicy.mx
}

Received 27 January 2011; Accepted 24 March 2011

Academic Editors: T. K. Basak and S. Cartmell

Copyright (๑) 2011 V. V. Zozulya. This is an open access article distributed under the Creative Commons Attribution License, which permits unrestricted use, distribution, and reproduction in any medium, provided the original work is properly cited.

Direct approach based on Betty's reciprocal theorem is employed to obtain a general formulation of Kirchhoff plate bending problems in terms of the boundary integral equation (BIE) method. For spatial discretization a collocation method with linear boundary elements (BEs) is adopted. Analytical formulas for regular and divergent integrals calculation are presented. Numerical calculations that illustrate effectiveness of the proposed approach have been done.

\section{Introduction}

The BIE method and its numerical realization BEM are powerful tools for analysis of the wide range problems in mechanics and engineering $[1,2]$. The main advantage of the BEM in comparison with other numerical methods (e.g., finite element method (FEM)) is that in the BEM only boundary of the domain and unknown functions on it have to be approximated. It leads to reduction of the problem dimension, that is, a $3 \mathrm{D}$ problem is transformed to a $2 \mathrm{D}$ one, and so forth. As result the amount of calculation is significantly reduced.

During the last decade, the BEM has been established as a robust numerical method for solution of the elastic thin plate problems [3]. Various aspects of the BEM application to the problems of thin Kirchhoff's plates bending have been reported in (see $[4-11,22]$, etc). In these and other publications calculations the corresponding integrals have been doing with application of numerical Gaussian's quadratures. It reduces accuracy and effectiveness of the BEM. In $[10,12]$ analytical integration has been applied, and exact formulas for the integrals evaluation have been obtained.

Another problem which gives many troubles in application of the BEM to the Kirchhoff's plates bending is the divergent integrals with singularities of the following type: weakly singular $O(\ln r)$, strongly singular $O\left(r^{-1}\right)$, and hypersingular $O\left(r^{-2}\right)$. For their calculation special methods have to be applied. Various methods for divergent integrals regularization have been developed in (see [13-16], etc). Application of the regularization algorithms for the divergent integrals evaluation in the BEM solution of the plate bending problems can be found in $[5,6,8,17]$. Information related to various aspects of the numerical solution of integral equations can be found in $[14,18,19]$ and related to integral calculation numerical using various quadrature formulas in $[20,21]$.

In this paper, we present a general direct BEM approach for thin Kirchhoff's plate bending problems. For evaluation of the regular integrals we use analytical formulas which give accurate results and need less time for calculation. For divergent integrals regularization we use special method developed in $[15,16]$. This method is based on theory of distribution and gives simple analytical equations for evaluation of weakly singular, strongly singular, and hypersingular integrals in the same way. Numerical examples that illustrate effectiveness of the developed BEM for calculation of the thin plate bending problems are presented.

\section{The Plate Equations}

Let us consider thin elastic plate with thickness $2 h$. The plate is subjected by load of the density $p(\mathbf{x})$, transversal to its 
middle surface $\Omega$, as it is shown on Figure 1. We suppose that deflection of the plate is small in comparison with its thickness, and Kirchhoff's hypothesis takes place [8].

We consider element that cuts out from the plate by two pairs of planes which are parallel to the planes $x_{1} x_{3}$ and $x_{2} x_{3}$ (Figure 2). Then equilibrium of the plate element can be expressed by the equations

$$
\begin{gathered}
\partial_{i} q_{i}(\mathbf{x})+\partial_{j} q_{j}(\mathbf{x})+p(\mathbf{x})=0, \\
\partial_{i} m_{i j}(\mathbf{x})-\partial_{j} m_{j}(\mathbf{x})+q_{j}(\mathbf{x})=0, \\
\partial_{j} m_{j i}(\mathbf{x})+\partial_{i} m_{i}(\mathbf{x})-q_{i}(\mathbf{x})=0 .
\end{gathered}
$$

Here and further on $w$ is a deflection of the plate, $m_{i j}$ are the bending and twisting moments, and $q_{i}(\mathbf{x})$ is a shear force. Indices $i, j$ possess the value 1,2 and correspond to coordinates $x_{1}, x_{2}$. Arrows on Figure 2 show positive directions of the bending and twisting moments.

From (1) follows inhomogeneous biharmonic differential equation for the deflection of the plate in the form [8]

$$
\Delta \Delta w=\frac{p}{D}, \quad \forall \mathbf{x} \in \Omega,
$$

where $\Delta \Delta$ is the biharmonic differential operator, $\Delta=\partial_{i} \partial_{i}$ is the Laplace operator, $\partial_{i}=\partial / \partial x_{i}$ is the partial derivative with respect to coordinate $x_{i}, v$ is Poisson's ratio, $\mathrm{D}=2 E h^{2} / 3(1-$ $\left.v^{2}\right)$ is the plate stiffness, and $E$ is the elastic modulus.

The plate deflection is related to other parameters of the plate by relations

$$
\begin{gathered}
\theta_{i}=\partial_{i} w, \quad m_{i, j}=-D\left[(1-v) \partial_{i} \partial_{j} w+v \delta_{i j} \partial_{k} \partial_{k} w\right\rfloor, \\
q_{i}=-D \partial_{i} \Delta w
\end{gathered}
$$

where $\theta_{i}$ is the slope of the element perpendicular to the middle surface of the plate.

Stress-strain state of the plate is defined by stress and strain tensors. Due to Kirchhoff's hypothesis they are related to the deflection by the equations

$$
\sigma_{i j}=-x_{3}\left(\lambda \delta_{i j} \Delta w+2 \mu \partial_{i} \partial_{j} w\right), \quad \varepsilon_{i j}=x_{3} \partial_{i} \partial_{j} w .
$$

Moments and shear force are expressed through stress tensor in the form

$$
m_{i j}=\int_{-h}^{h} \sigma_{i j} x_{3} d x_{3}, \quad q_{i}=\int_{-h}^{h} \sigma_{i 3} x_{3} d x_{3}, \quad(i=1,2) .
$$

Generalized shear force may be introduced by the relation

$$
v_{n}=q_{n}-\partial_{t} m_{t} .
$$

Correct statement of the plate bending problem supposes that (2) is supplemented by suitable conditions on the boundary $\partial \Omega$. In the Kirchhoff's theory of plates boundary conditions are usually prescribed to the functions [8]

$$
\begin{gathered}
w, \quad \theta_{n}=\theta_{i} n_{i}=\partial_{n} w, \\
m_{n}=m_{i j} n_{i} n_{j}=-D\left(\partial_{n} \partial_{n} w+\nu \partial_{t} \partial_{t} w\right), \\
v_{n}=q_{i} n_{i}-\partial_{t}\left(m_{i j} n_{i} t_{j}\right)=-D \partial_{n}\left[\Delta w+(1-\nu) \partial_{t}^{2} w\right],
\end{gathered}
$$

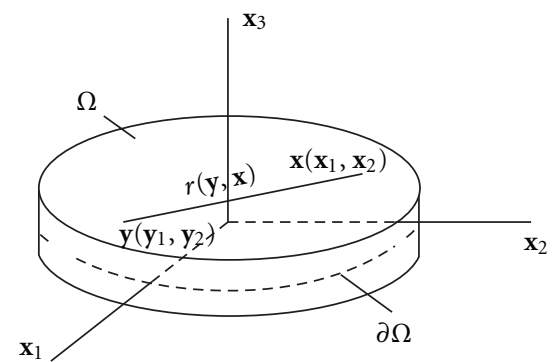

Figure 1: Bending of a thin plate.

where $n_{i} u t_{i}$ are the components of vectors normal and tangential to the boundary $\partial \Omega$, respectively, $\partial_{n}=n_{i} \partial_{i}$ and $\partial_{t}=t_{i} \partial_{i}$ are normal and tangential derivatives, respectively.

For arbitrary part of the boundary we have (see Figure 3)

$$
\begin{gathered}
m_{i}=m_{i j} n_{j}, \quad m_{n}=m_{i} n_{i}=m_{i j} n_{i} n_{j}, \quad q_{i}=q_{i} n_{i}, \\
m_{t}=m_{i} t_{i}=m_{i j} n_{i} t_{j}, \quad m_{i}=m_{n} n_{i}+m_{t} t_{i}, \\
\theta_{t i}=\theta_{i} t_{i}, \quad \theta_{i}=\theta_{n} n_{i}+\theta_{t} t_{i} .
\end{gathered}
$$

In the case if boundary contains corner points, corner forces $q_{c}$ which are generated by $m_{t}$ in the points where tangential vector has break have to be taken into account in the expression for $v_{n}$. Really from the Figure 4 follows that the $m_{t}$ acts on different sides of any angle $\alpha$ in opposite directions and $m_{t}^{-}$and $m_{t}^{+}$related to the different sides of the angle therefore, $q_{c}=m_{t}^{-}-m_{t}^{+}$.

Under the Kirchhoff assumptions boundary conditions can be defined as follows:

$$
\begin{array}{ccc}
w=0, & \theta_{n}=0, & \text { clamped contour }\left(q_{c}=0, \quad w_{c}=0\right) \\
w=0, & m_{n}=0, & \text { simply-supported contour } \\
& \left(q_{c} \neq 0, w_{c}=0\right) \\
v_{n}=0, & m_{n}=0, & \text { free contour }\left(q_{c}=0, w_{c} \neq 0\right)
\end{array}
$$

Each of the boundary conditions (9)-(11) together with differential equation for the deflection of the plate (2) represents corresponding boundary value problem.

\section{Betty's Reciprocal Theorem and Elastic Potentials}

The boundary-value problems (2), (9)-(11) can be solved by the BIE method. In order to transform a boundary-value problem to the BIE, Betty's reciprocal theorem can be used in the following form $[1,3]$ :

$$
\begin{aligned}
\int_{\Omega} p w^{\prime} d \Omega+\int_{\partial \Omega}\left(q_{n} w^{\prime}+m_{i} \theta_{i}^{\prime}\right) d S \\
=\int_{\Omega} p^{\prime} w d \Omega+\int_{\partial \Omega}\left(q_{n}^{\prime} w+m_{i}^{\prime} \theta_{i}\right) d S .
\end{aligned}
$$



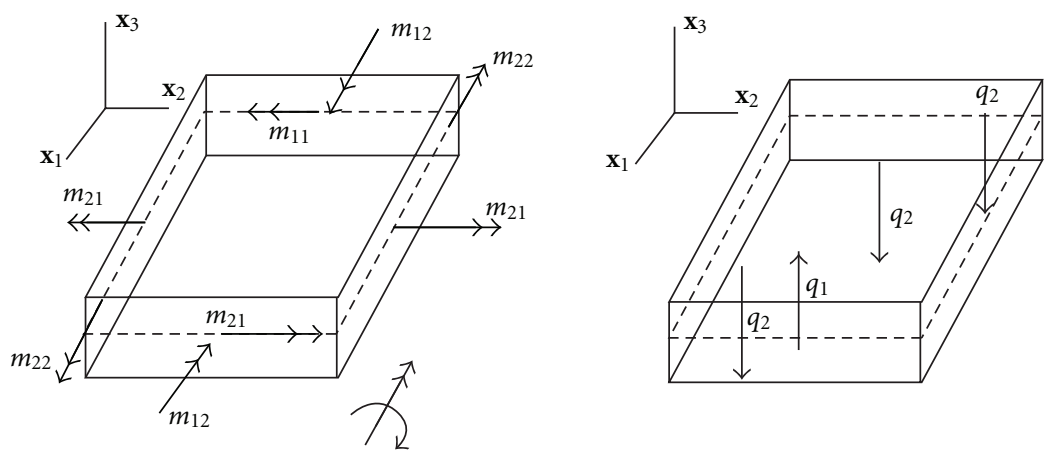

Figure 2: Elements of the bending plate.
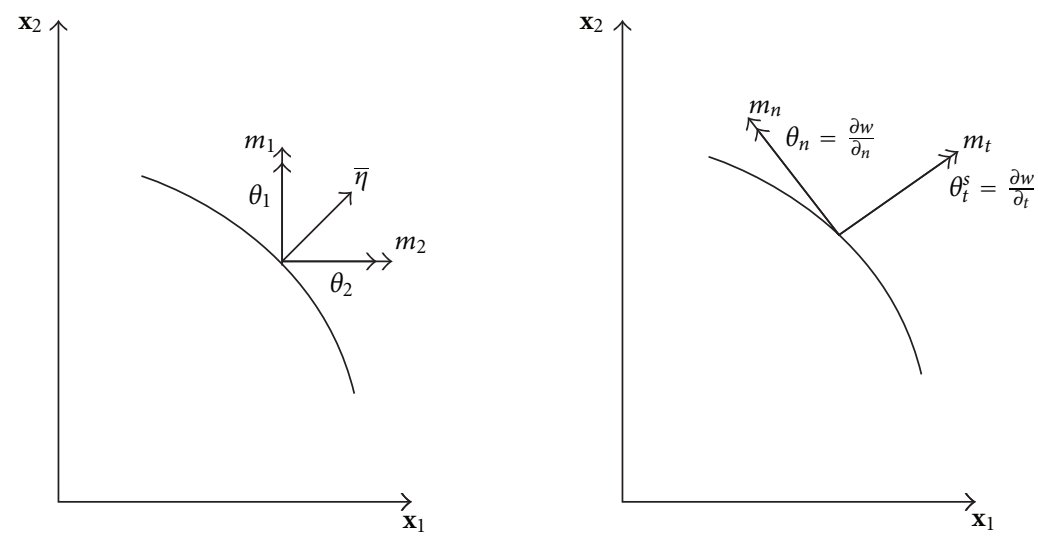

FIGURE 3: Arbitrary part of the boundary.

In expression (12) functions with accent $p^{\prime}, w^{\prime}, \theta_{n}^{\prime}, m_{n}^{\prime}, v_{n}^{\prime}$ and without accent $p, w, \theta_{n}, m_{n}, v_{n}$ are parameters of two different states of the elastic plate, respectively.

Let us transform (12) to the form suitable for the case of presence mentioned above corner points. For that purpose we consider series of integral transformations

$$
\begin{aligned}
\int_{\Omega} m_{i} \theta_{i}^{\prime} d S & =\int_{\partial \Omega}\left(m_{n} n_{i}+m_{t} t_{i}\right)\left(\theta_{n}^{\prime} n_{i}+\theta_{t}^{\prime} t_{i}\right) d S \\
& =\int_{\partial \Omega}\left(m_{n} \theta_{n}^{\prime}+m_{t} \theta_{t}^{\prime}\right) d S \\
& =\int_{\partial \Omega}\left(m_{n} \theta_{n}^{\prime}+m_{t} \partial_{t} w^{\prime}\right) d S .
\end{aligned}
$$

Last integral in (13) can be represented in the form

$$
\int_{\partial \Omega} m_{t} \partial_{t} w^{\prime} d S=\int_{\partial \Omega} \partial_{t}\left(m_{t} w^{\prime}\right) d S-\int_{\partial \Omega} \partial_{t} m_{t} w^{\prime} d S .
$$

For close contour the first right integral in (14) is equal to

$$
I=\int_{\partial \Omega} \partial_{t}\left(m_{t} w^{\prime}\right) d S=\int_{\partial \Omega} d\left(m_{t} w^{\prime}\right) d S=0 .
$$

For contour with corner points

$$
I=m_{t}^{-} w^{\prime}-m_{t}^{+} w=\left(m_{t}^{-}-m_{t}^{+}\right) w=q_{c} w_{c}^{\prime} .
$$

Finally integral (13) can be presented in the form

$$
\int_{\partial \Omega} m_{i} \theta_{i}^{\prime} d S=\int_{\partial \Omega}\left(m_{n} \theta_{n}^{\prime}-\partial_{t} m_{t} w^{\prime}\right) d S+q_{c} w_{c}^{\prime} .
$$

In the same way can be transformed integral

$$
\int_{\partial \Omega} m_{i}^{\prime} \theta_{i} d S=\int_{\partial \Omega}\left(m_{n}^{\prime} \theta_{n}-\partial_{t} m_{t}^{\prime} w\right) d S+q_{c}^{\prime} w_{c} .
$$

With taking into account representations (17) and (18) Betty's reciprocal relation (12) can be extended for the case of the corner points presence and can be represented in the form

$$
\begin{aligned}
\int_{\Omega} p w^{\prime} d \Omega+\int_{\partial \Omega}\left(v_{n} w^{\prime}+m_{n} \theta_{n}^{\prime}\right) d S+q_{c} w_{c}^{\prime} \\
=\int_{\Omega} p^{\prime} w d \Omega+\int_{\partial \Omega}\left(v_{n}^{\prime} w+m_{n}^{\prime} \theta_{n}\right) d S+q_{c}^{\prime} w_{c} .
\end{aligned}
$$

From Betty's reciprocal relation (13) one can obtain Somigliano's type identity for the deflection of the plate $w$. To do that we choose as the first state of plate (without accent) that state, which has to be calculated. The second state of the plate (with accent) is auxiliary and correspond to infinite plate subjected to action of the concentrated force of unit density that applied at point $\mathbf{x}$ in the direction normal to the middle surface. Functions that correspond to the second state 

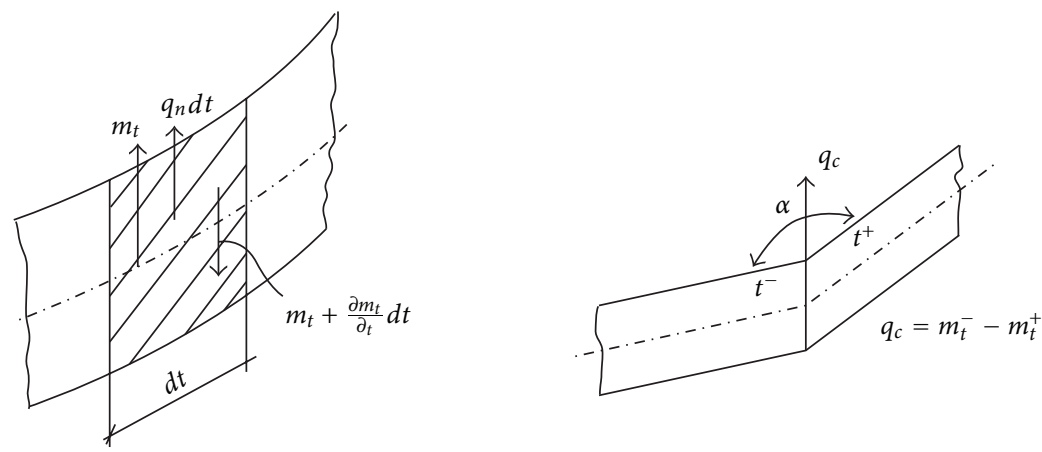

FIGURE 4: Action of the generalized shear force on the corner pint.

are fundamental solutions for the plate. For convenience the following notations will be used:

$$
\begin{gathered}
p^{\prime}=\delta(\mathbf{y}, \mathbf{x}), \quad w^{\prime}=W(\mathbf{y}, \mathbf{x}), \quad \theta_{n}^{\prime}=\Theta(\mathbf{y}, \mathbf{x}), \\
m_{n}^{\prime}=M(\mathbf{y}, \mathbf{x}), \quad v_{n}^{\prime}=V(\mathbf{y}, \mathbf{x}) .
\end{gathered}
$$

Taking into account that Dirac's delta function $\delta(\mathbf{x}, \mathbf{y})$ transform regular functions in the following way:

$$
\int_{\Omega} w(\mathbf{y}) \delta(\mathbf{y}, \mathbf{x}) d \Omega=w(\mathbf{x}),
$$

Betty's reciprocal relation (19) can be transformed to the Somigliano's type identity for the plate deflection

$$
\begin{aligned}
& w(\mathbf{x})=\int_{\partial \Omega}\left[v_{n}(\mathbf{y}) W(\mathbf{y}, \mathbf{x})+m_{n}(\mathbf{y}) \Theta(\mathbf{y}, \mathbf{x})\right. \\
&\left.-\theta_{n}(\mathbf{y}) M(\mathbf{y}, \mathbf{x})-w(\mathbf{y}) V(\mathbf{y}, \mathbf{x})\right] d S \\
&+\int_{\Omega} p(\mathbf{y}) W(\mathbf{y}, \mathbf{x}) d \Omega \\
&+ \sum_{k=1}^{K}\left(q_{c} W^{c}(\mathbf{y}, \mathbf{x})-M^{c}(\mathbf{y}, \mathbf{x}) w_{c}\right)_{k}, \\
& \forall \mathbf{x} \in \mathrm{R}^{2} \backslash \partial \Omega .
\end{aligned}
$$

Here $K$ is the number of corner points on the contour of the plate $\partial \Omega$.

The slope of the element perpendicular to the middle surface of the plate $\theta_{n}(\mathbf{x})$ can be obtained applying operator of the normal derivative $\partial_{n}$ to (22). We assume that point $\mathbf{x} \in R^{2} \backslash \partial \Omega$ and therefore kernels in (22) are sufficiently smooth and differentiation with respect to parameter $\mathbf{x}$ is correct. As result we obtain

$$
\begin{aligned}
& \theta_{n}(\mathbf{x})=\int_{\partial \Omega}\left[v_{n}(\mathbf{y}) W_{n}(\mathbf{y}, \mathbf{x})+m_{n}(\mathbf{y}) \Theta_{n}(\mathbf{y}, \mathbf{x})\right.\left.-\theta_{n}(\mathbf{y}) M_{n}(\mathbf{y}, \mathbf{x})-w(\mathbf{y}) V_{n}(\mathbf{y}, \mathbf{x})\right] d S \\
&+\int_{\Omega} p(\mathbf{y}) W_{n}(\mathbf{y}, \mathbf{x}) d \Omega \\
&+\sum_{k=1}^{K}\left(q_{c} W_{n}^{c}(\mathbf{y}, \mathbf{x})-M_{n}^{c}(\mathbf{y}, \mathbf{x}) \partial_{n} w_{c}\right)_{k}, \\
& \forall \mathbf{x} \in R^{2} \backslash \partial \Omega .
\end{aligned}
$$

The kernels $W_{n}, \Theta_{n}, M_{n}$, and $V_{n}$ can be obtained by applying operator of the normal derivative $\partial_{n}$ with respect to $\mathbf{x}$ to corresponding fundamental solutions in (22).

Further in order to construct BIE limit transition to the boundary $\mathbf{x} \rightarrow \partial \Omega$ will be done.

For convenience and compactness of the BIE consideration we introduce the following contour and area potentials:

$$
\begin{aligned}
W\left(v_{n}, \mathbf{x}, \partial \Omega\right) & =\int_{\partial \Omega} v_{n}(\mathbf{y}) W(\mathbf{y}, \mathbf{x}) d S, \\
\Theta\left(m_{n}, \mathbf{x}, \partial \Omega\right) & =\int_{\partial \Omega} m_{n}(\mathbf{y}) \Theta(\mathbf{y}, \mathbf{x}) d S, \\
M\left(\theta_{n}, \mathbf{x}, \partial \Omega\right) & =\int_{\partial \Omega} \theta_{n}(\mathbf{y}) M(\mathbf{y}, \mathbf{x}) d S, \\
V(w, \mathbf{x}, \partial \Omega) & =\int_{\partial \Omega} w(\mathbf{y}) V(\mathbf{y}, \mathbf{x}) d S, \\
W_{n}\left(v_{n}, \mathbf{x}, \partial \Omega\right) & =\int_{\partial \Omega} v_{n}(\mathbf{y}) W_{n}(\mathbf{y}, \mathbf{x}) d S, \\
\Theta_{n}\left(m_{n}, \mathbf{x}, \partial \Omega\right) & =\int_{\partial \Omega} m_{n}(\mathbf{y}) \Theta_{n}(\mathbf{y}, \mathbf{x}) d S, \\
M_{n}\left(\theta_{n}, \mathbf{x}, \partial \Omega\right) & =\int_{\partial \Omega} \theta_{n}(\mathbf{y}) M_{n}(\mathbf{y}, \mathbf{x}) d S, \\
V_{n}(w, \mathbf{x}, \partial \Omega) & =\int_{\partial \Omega} w(\mathbf{y}) V_{n}(\mathbf{y}, \mathbf{x}) d S, \\
W(p, \mathbf{x}, \Omega) & =\int_{\Omega} p(\mathbf{y}) W(\mathbf{y}, \mathbf{x}) d \Omega, \\
W_{n}(p, \mathbf{x}, \Omega) & =\int_{\Omega} p(\mathbf{y}) W_{n}(\mathbf{y}, \mathbf{x}) d \Omega .
\end{aligned}
$$

Now with taking into account (23) и (25) integral representations, (22) и (23) can be written in the form

$$
\begin{aligned}
w(\mathbf{x})= & W\left(v_{n}, \mathbf{x}, \partial \Omega\right)+\Theta\left(m_{n}, \mathbf{x}, \partial \Omega\right)-M\left(\theta_{n}, \mathbf{x}, \partial \Omega\right) \\
& -V(w, \mathbf{x}, \partial \Omega)+W(p, \mathbf{x}, \Omega) \\
& +\sum_{k=1}^{K}\left(q_{c} W^{c}(\mathbf{y}, \mathbf{x})-M^{c}(\mathbf{y}, \mathbf{x}) w_{c}\right)_{k},
\end{aligned}
$$




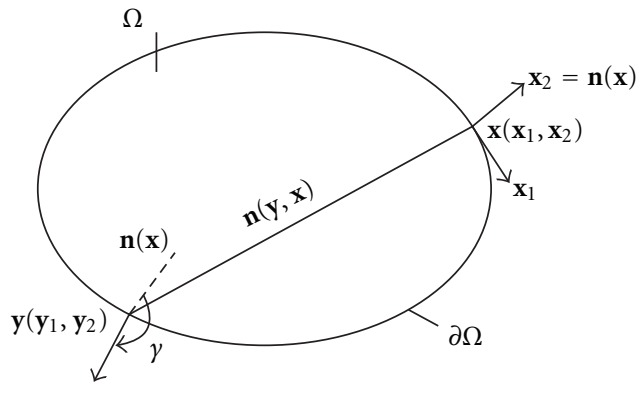

Figure 5

$$
\begin{gathered}
\forall \mathbf{x} \in \mathrm{R}^{2} \backslash \partial \Omega \\
\theta_{n}(\mathbf{x})=W_{n}\left(v_{n}, \mathbf{x}, \partial \Omega\right)+\Theta_{n}\left(m_{n}, \mathbf{x}, \partial \Omega\right)-M_{n}\left(\theta_{n}, \mathbf{x}, \partial \Omega\right) \\
-V_{n}(w, \mathbf{x}, \partial \Omega)+W_{n}(p, \mathbf{x}, \Omega) \\
+\sum_{k=1}^{K}\left(q_{c} W_{n}^{c}(\mathbf{y}, \mathbf{x})-M_{n}^{c}(\mathbf{y}, \mathbf{x}) \partial_{n} w_{c}\right)_{k}, \\
\forall \mathbf{x} \in \mathrm{R}^{2} \backslash \partial \Omega .
\end{gathered}
$$

\section{Fundamental Solutions}

Fundamental solution $W(\mathbf{y}, \mathbf{x})$ for static plate bending is the solution of the differential equation

$$
D \Delta \Delta(\mathbf{y}) W(\mathbf{y}, \mathbf{x})=\delta(\mathbf{y}, \mathbf{x}), \quad \forall \mathbf{x} \in \mathrm{R}^{2},
$$

where $\Delta \Delta(\mathbf{y})$ is the biharmonic differential operator with respect to $\mathbf{y}\left(y_{1}, y_{2}\right)$ defined in (2).

Solution of (27) has the form

$$
W(\mathbf{y}, \mathbf{x})=\frac{1}{8 \pi D} r^{2} \ln r
$$

where $r=\sqrt{\left(y_{1}-x_{1}\right)^{2}+\left(y_{2}-x_{2}\right)^{2}}$ is the distance between points $\mathbf{y}$ and $\mathbf{x}$

Fundamental solutions $\Theta(\mathbf{y}, \mathbf{x}), M(\mathbf{y}, \mathbf{x})$ and $V(\mathbf{y}, \mathbf{x})$ from the integral representation (22) can be calculated applying differential operators (3) to $W(\mathbf{y}, \mathbf{x})$ with taking into account (6) and direction of the unit vector $\mathbf{n}(\mathbf{y})$ (see Figure 5).

The fundamental solutions $\Theta(\mathbf{y}, \mathbf{x}), M(\mathbf{y}, \mathbf{x}), V(\mathbf{y}, \mathbf{x})$ have the form

$$
\begin{gathered}
\Theta(\mathbf{y}, \mathbf{x})=-\frac{r}{8 \pi D} \frac{\partial r}{\partial n}(1+2 \ln r), \\
M(\mathbf{y}, \mathbf{x})=-\frac{1}{8 \pi}\left[2\left(\frac{\partial^{2} r}{\partial n^{2}}+\nu \frac{\partial^{2} r}{\partial t^{2}}\right)+(1+v)(1+2 \ln r)\right], \\
V(\mathbf{y}, \mathbf{x})=\frac{r}{4 \pi} \frac{\partial r}{\partial n}\left[5-v-2\left(\frac{\partial^{2} r}{\partial n^{2}}+(2-v) \frac{\partial^{2} r}{\partial t^{2}}\right)\right] .
\end{gathered}
$$

Fundamental solutions $W_{n}(\mathbf{y}, \mathbf{x}), \Theta_{n}(\mathbf{y}, \mathbf{x}), M_{n}(\mathbf{y}, \mathbf{x})$, and $V_{n}(\mathbf{y}, \mathbf{x})$, from the integral representation (23) can be calculated applying operator of the normal derivative $\partial_{n}$ with respect to $\mathbf{x}$ to $W(\mathbf{y}, \mathbf{x}), \Theta(\mathbf{y}, \mathbf{x}), M(\mathbf{y}, \mathbf{x})$, and $V(\mathbf{y}, \mathbf{x})$ from (28) and (29) with taking into direction of the unit vector $\mathbf{n}(\mathbf{x})$ (Figure 5$)$.

Finally the fundamental solutions $W_{n}(\mathbf{y}, \mathbf{x}), \Theta_{n}(\mathbf{y}, \mathbf{x})$, $M_{n}(\mathbf{y}, \mathbf{x})$, and $V_{n}(\mathbf{y}, \mathbf{x})$ can be represented in the form

$$
\begin{aligned}
W_{n}(\mathbf{y}, \mathbf{x})= & -\frac{y_{2}}{8 \pi D}(1+2 \ln r), \\
\Theta_{n}(\mathbf{y}, \mathbf{x})=- & \frac{1}{8 \pi D}\left(\frac{2 y_{2}}{r} \frac{\partial r}{\partial n}-(1+2 \ln r) \cos \gamma\right), \\
M_{n}(\mathbf{y}, \mathbf{x})=-\frac{1}{4 \pi r^{2}}\left[2 r\left(\frac{\partial r}{\partial n} \cos \gamma-\nu \frac{\partial r}{\partial t} \sin \gamma\right)\right. & \left.-y_{2}(1+\nu)+2 y_{2}\left(\frac{\partial^{2} r}{\partial n^{2}}+\nu \frac{\partial^{2} r}{\partial t^{2}}\right)\right], \\
V_{n}(\mathbf{y}, \mathbf{x}) \begin{aligned}
1 \\
=
\end{aligned} & (\nu-5)\left(r \cos \gamma+2 y_{2} \frac{\partial r}{\partial n} \sin \gamma\right) \\
& \times 6 r \frac{\partial^{2} r}{\partial n^{2}} \cos \gamma+8 y_{2} \frac{\partial r}{\partial n} \\
& \times\left(\frac{\partial^{2} r}{\partial n^{2}}+(2-v) \frac{\partial^{2} r}{\partial t^{2}}\right) \\
+ & \left.2 r(2-v)+\frac{\partial r}{\partial t}\left(\frac{\partial r}{\partial t} \cos \gamma-2 \frac{\partial r}{\partial n} \sin \gamma\right)\right] .
\end{aligned}
$$

Here $\gamma$ is the angle between normal vector $\mathbf{n}(\mathbf{x})$ and axis $y_{2}$, counted from $\mathbf{n}(\mathbf{x})$ clockwise.

Analysis of (28)-(30) shows that with $\mathbf{x} \rightarrow \mathbf{y}$

$$
\begin{aligned}
W(\mathbf{y}, \mathbf{x}) \longrightarrow r^{2} \ln r, & \Theta(\mathbf{y}, \mathbf{x}) \longrightarrow r \ln r, \\
M(\mathbf{y}, \mathbf{x}) \longrightarrow \ln r, & V(\mathbf{y}, \mathbf{x}) \longrightarrow r^{-1}, \\
W_{n}(\mathbf{y}, \mathbf{x}) \longrightarrow r \ln r, & \Theta_{n}(\mathbf{y}, \mathbf{x}) \longrightarrow \ln r, \\
M_{n}(\mathbf{y}, \mathbf{x}) \longrightarrow r^{-1}, & V_{n}(\mathbf{y}, \mathbf{x}) \longrightarrow r^{-2} .
\end{aligned}
$$

From (31) it follows that for $\mathbf{x} \rightarrow \mathbf{y}$ kernels $W(\mathbf{y}, \mathbf{x}), \Theta(\mathbf{y}, \mathbf{x})$ and $W_{n}(\mathbf{y}, \mathbf{x})$ are continuous, kernels $M(\mathbf{y}, \mathbf{x}) и \Theta_{n}(\mathbf{y}, \mathbf{x})$ are weakly singular, kernels $V(\mathbf{y}, \mathbf{x}) и M_{n}(\mathbf{y}, \mathbf{x})$ are strongly singular, and kernel $V_{n}(\mathbf{y}, \mathbf{x})$ is hypersingular. Potentials with continuous kernels $W\left(v_{n}, \mathbf{x}, \partial \Omega\right), \Theta\left(m_{n}, \mathbf{x}, \partial \Omega\right)$, and $W_{n}\left(v_{n}, \mathbf{x}, \partial \Omega\right)$ can be considered in usual (Riemann or Lebegue) sense, potentials with weakly singular kernels $M\left(\theta_{n}, \mathbf{x}, \partial \Omega\right)$ and $\Theta_{n}\left(m_{n}, \mathbf{x}, \partial \Omega\right)$ can be considered as improper, potentials with strongly singular kernels $V(w, \mathbf{x}, \partial \Omega)$, and $M_{n}\left(\theta_{n}, \mathbf{x}, \partial \Omega\right)$ have to be considered in the sense of Cauchy principal values and potential with hypersingular kernel in sense of Hadamard's finite part $V_{n}(w, \mathbf{x}, \partial \Omega)($ see $[2,5,6,19,21])$.

For $\mathbf{x} \rightarrow \partial \Omega$ above-mentioned singularities may arise in the boundary potentials (24) and therefore not all of them cross the boundary continuously. Boundary properties of these potentials have been well studied in (see $[1,3]$, etc). 
Therefore, only final results will be discussed here. These are expressed by the equations

$$
\begin{gathered}
W\left(v_{n}, \mathbf{x}, \partial \Omega\right)^{ \pm}=W\left(v_{n}, \mathbf{x}, \partial \Omega\right)^{0}, \\
\Theta\left(m_{n}, \mathbf{x}, \partial \Omega\right)^{ \pm}=\Theta\left(m_{n}, \mathbf{x}, \partial \Omega\right)^{0}, \\
M\left(\theta_{n}, \mathbf{x}, \partial \Omega\right)^{ \pm}=M\left(\theta_{n}, \mathbf{x}, \partial \Omega\right)^{0}, \\
V(w, \mathbf{x}, \partial \Omega)^{ \pm}=\mp \frac{1}{2} w(\mathbf{x})+V(w, \mathbf{x}, \partial \Omega)^{0}, \\
W_{n}\left(v_{n}, \mathbf{x}, \partial \Omega\right)^{ \pm}=W_{n}\left(v_{n}, \mathbf{x}, \partial \Omega\right)^{0}, \\
\Theta_{n}\left(m_{n}, \mathbf{x}, \partial \Omega\right)^{ \pm}=\Theta_{n}\left(m_{n}, \mathbf{x}, \partial \Omega\right)^{0}, \\
M_{n}\left(\theta_{n}, \mathbf{x}, \partial \Omega\right)^{ \pm}= \pm \frac{1}{2} \theta_{n}+M_{n}\left(\theta_{n}, \mathbf{x}, \partial \Omega\right)^{0} \\
V_{n}(w, \mathbf{x}, \partial \Omega)^{ \pm}=V(w, \mathbf{x}, \partial \Omega)^{0} .
\end{gathered}
$$

Here symbols " \pm " and “ $\mp$ " denote that two equalities, one with upper and the other with lower signs, are considered. The sign " 0 " indicates that the direct value of the corresponding potentials on the boundary $\partial \Omega$ should be taken.

\section{Boundary Integral Equations for Boundary-Value Problems}

In order to get integral representations for the deflection of the plate $w(\mathbf{x})$ and slope of the element perpendicular to the middle surface of the plate $\theta_{n}(\mathbf{x})$ on the boundary $\partial \Omega$ limit transition $\mathbf{x} \rightarrow \partial \Omega$ has to be done. With taking into account boundary properties of the elastic potentials (32) on smooth parts of the boundary the following equations can be obtained:

$$
\begin{aligned}
\frac{1}{2} w(\mathbf{x})= & W\left(v_{n}, \mathbf{x}, \partial \Omega\right)+\Theta\left(m_{n}, \mathbf{x}, \partial \Omega\right)-M\left(\theta_{n}, \mathbf{x}, \partial \Omega\right) \\
& -V(w, \mathbf{x}, \partial \Omega)+W(p, \mathbf{x}, \Omega), \quad \forall \mathbf{x} \in \partial \Omega, \\
\frac{1}{2} \theta_{n}(\mathbf{x})= & W_{n}\left(v_{n}, \mathbf{x}, \partial \Omega\right)+\Theta_{n}\left(m_{n}, \mathbf{x}, \partial \Omega\right)-M_{n}\left(\theta_{n}, \mathbf{x}, \partial \Omega\right) \\
& -V_{n}(w, \mathbf{x}, \partial \Omega)+W_{n}(p, \mathbf{x}, \Omega), \quad \forall \mathbf{x} \in \partial \Omega .
\end{aligned}
$$

For simplicity in this section we did not take into account corner point.

For any boundary-value problem (2), (9)-(11) can be constructed corresponding BIE based on boundary integral representations (33). Several examples of the BIE that correspond to main boundary-value problems for plates are presented bellow.

Boundary-Value Problem (2), (9)

One has

$$
\begin{array}{r}
W\left(v_{n}, \mathbf{x}, \partial \Omega\right)+\Theta\left(m_{n}, \mathbf{x}, \partial \Omega\right)+W(p, \mathbf{x}, \Omega)=0, \\
\forall \mathbf{x} \in \partial \Omega \\
W_{n}\left(v_{n}, \mathbf{x}, \partial \Omega\right)+\Theta_{n}\left(m_{n}, \mathbf{x}, \partial \Omega\right)+W_{n}(p, \mathbf{x}, \Omega)=0, \\
\forall \mathbf{x} \in \partial \Omega .
\end{array}
$$

This system of the BIE is the system of Fredholm integral equations of the first kind with smooth kernels. Such integral equations correspond the so-called ill-posed problems [19]. Different kind of instability can occur during their numerical solution. For their correct solution special regularization technique has to be applied [19].

Boundary Value Problem (2), (10)

One has

$$
\begin{aligned}
& W\left(v_{n}, \mathbf{x}, \partial \Omega\right)+M\left(\theta_{n}, \mathbf{x}, \partial \Omega\right)+W(p, \mathbf{x}, \Omega)=0, \\
& \forall \mathbf{x} \in \partial \Omega \\
& W_{n}\left(v_{n}, \mathbf{x}, \partial \Omega\right)-\frac{1}{2} \theta_{n}(\mathbf{x})-M_{n}\left(\theta_{n}, \mathbf{x}, \partial \Omega\right)+W_{n}(p, \mathbf{x}, \Omega)=0, \\
& \forall \mathbf{x} \in \partial \Omega .
\end{aligned}
$$

This system of the BIE consists of the Fredholm integral equation of the first kind with smooth kernels and singular integral equation. Specific features of the Fredholm integral equation solution have been considered above. Singular integral equations can be solved using technique developed in [14] of the divergent integrals regularization. In process of the system of integral equations (35) solution specific features of both equations have to be taken into account.

Boundary Value Problem (2), (10)

One has

$$
\begin{array}{r}
\frac{1}{2} w(\mathbf{x})+V(w, \mathbf{x}, \partial \Omega)+M\left(\theta_{n}, \mathbf{x}, \partial \Omega\right)=W(p, \mathbf{x}, \Omega), \\
\forall \mathbf{x} \in \partial \Omega_{c} \\
\frac{1}{2} \theta_{n}(\mathbf{x})+M_{n}\left(\theta_{n}, \mathbf{x}, \partial \Omega\right)+V_{n}(w, \mathbf{x}, \partial \Omega)=W_{n}(p, \mathbf{x}, \Omega), \\
\forall \mathbf{x} \in \partial \Omega_{c} .
\end{array}
$$

Integral operations in this system of integral equations contain kernels with different singularities. Integrals with logarithmic singularity $\ln r$ are weakly singular, they can be consider as improper. Integrals with singularity $r^{-1}$ are strongly singular and have to be considered in the sense of Cauchy principle value. Integrals with singularity $r^{-2}$ are hypersingular and have to be considered in the sense of Hadamard's finite part. For more information about divergent integrals and their application in BIE one can refer to $[5,6,8,13-16]$ and references here.

In each specific case corresponding system of BIE can be easily constructed based on (34)-(36).

\section{BEM and Approximation of the Region and Functions}

The BEM can be treated as the approximate method for the BIE solution, which includes approximation of the functions and the domain where they are defined by discrete finite dimensional model. Let us construct discrete model the plate boundary $\partial \Omega \in R$. We fix in the $\partial \Omega$ finite number of points 


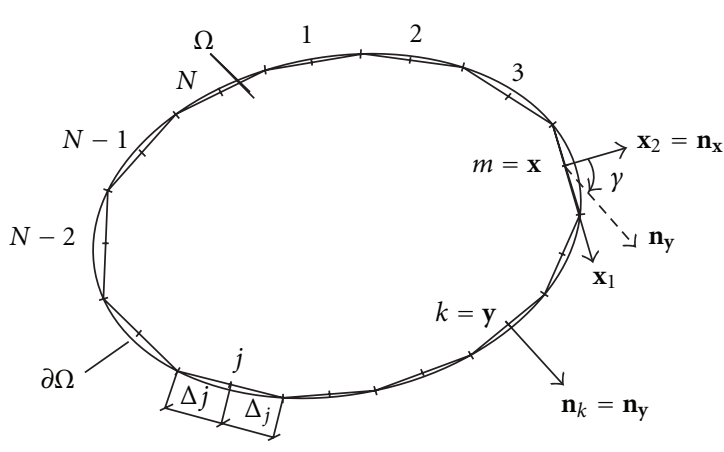

Figure 6: Linear BE.

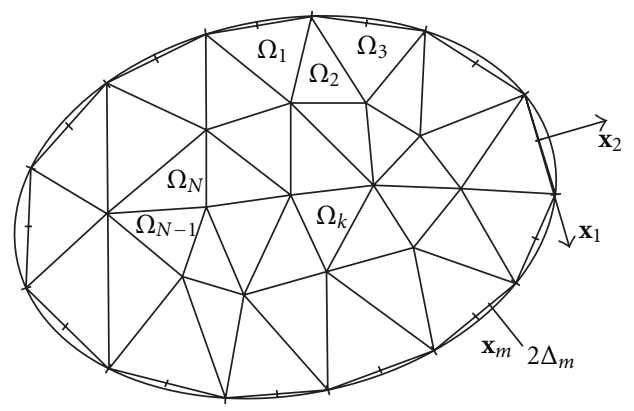

FIgURE 7: Discretization of the domain $\Omega$.

$\mathbf{x}_{g}(g=1, \ldots, G)$. These points are refered to as global nodes points $V(g)=\left\{x_{g} \in \partial \Omega: g=1, \ldots, G\right\}$.

We shall divide the boundary $\partial \Omega$ into finite number of subdomains $\partial \Omega_{n}(n=1, \ldots, N)$, such that they satisfy the following conditions:

$$
\partial \Omega_{m} \cap \partial \Omega_{k}=\varnothing, \quad(\text { если } m \neq k), \partial \Omega=\bigcup_{k=1}^{N} \partial \Omega_{k} .
$$

On each FE we introduce a local coordinate system $\xi$. We designate the nodal points $\mathbf{x}^{q} \in V_{n}$ in the local system of coordinates by $\xi^{q}$. They are coordinates of the nodal points in the local coordinate system. Local and global coordinate are related in the following way:

$$
\mathbf{x}^{q}=\sum_{n=1}^{N} \Lambda_{n} \xi_{n}^{q}
$$

Functions $\boldsymbol{\Lambda}_{n}$ depend on position of the nodal points in the BE. They join individual BE together in the BE model. Borders of the BEs and position of the nodal points should be such that, after joining together, separate elements form discrete model of the plate boundary $\partial \Omega$.

Having constructed BE model of the area $\Omega$, we can consider approximation of the function $f(\mathbf{x})$ that belong to some functional space. The BE model of the boundary $\partial \Omega$ is the domain of function which should be approximated. We denote function $f(\mathbf{x})$ on the $\mathrm{BE} \Omega_{n}$ by $f^{n}(\mathbf{x})$. Then

$$
f(\mathbf{x})=\sum_{n=1}^{N} f^{n}(\mathbf{x}) .
$$

On each BE the local functions $f^{n}(\mathbf{x})$ may be represented in the form

$$
f^{n}(\mathbf{x}) \approx \sum_{q=1}^{Q} f^{n}\left(\mathbf{x}^{q}\right) \varphi_{n q}(\xi),
$$

where $\varphi_{n q}(\xi)$ are interpolation polynomials or shape functions on the $\mathrm{BE}$ with number $n$. In nodal point with coordinates $\mathbf{x}^{q}$ they are equal to 1 and in other nodal points are equal to zero. Taking into account (39) and (40) global approximation of the function $f(\mathbf{x})$ looks like

$$
f(\mathbf{x}) \approx \sum_{n=1}^{N} \sum_{q=1}^{Q} f^{n}\left(\mathbf{x}_{q}\right) \varphi_{n q}(\xi) .
$$

If the nodal point $q$ belongs to several FEs it is considered in these sums only once.

In general case BEs can be of different shape and size with different shape functions defined on them. The simplest are linear BE with piecewise constant shape functions as it is shown in Figure 6.

For linear $\mathrm{BE}$ and piecewise constant shape functions corresponding integrals can be calculated analytically. It can significantly simplify calculations, reduce time, and increase accuracy and stability of the calculation process. Advantages of application curvilinear BE and high-order shape functions consist in more accurate approximation of the boundary and functions, but it leads to complication of calculations. Some time that circumstance can devalue the above-mentioned advantages. For more information regarding advantages and disadvantages of different BEs and function approximation refer to $[1,2]$. Taking into account all the above we use here the linear $\mathrm{BE}$ and the piecewise constant shape functions.

Let us divide boundary $\partial \Omega$ into $N$ curvilinear $\mathrm{BE} \partial \Omega_{n}$, which satisfy conditions (37). Replacing curvilinear BE by linear ones as it is shown in Figure 6, we obtain discrete approximation of the plate boundary by linear segments of the length $2 \Delta_{n}$. In accordance with model that has been used here $w(\mathbf{x}), \theta_{n}(\mathbf{x}), m_{n}(\mathbf{x})$ и $v_{n}(\mathbf{x})$ are constant on each BE.

In order to calculate integrals over domain in (25) we divide domain $\Omega$ into $L$ triangular elements as it is shown in Figure 7.

Therefore, finite dimensional system of the BEM equations has the form

$$
\begin{aligned}
\frac{1}{2} w\left(\mathbf{x}_{m}\right)=\sum_{k=1}^{N}[ & v_{n}\left(\mathbf{y}_{k}\right) W\left(\mathbf{x}_{m} \mathbf{y}_{k}\right)+m_{n}\left(\mathbf{y}_{k}\right) \Theta\left(\mathbf{x}_{m} \mathbf{y}_{k}\right) \\
& \left.-\theta_{n}\left(\mathbf{y}_{k}\right) M\left(\mathbf{x}_{m} \mathbf{y}_{k}\right)-w\left(\mathbf{y}_{k}\right) V\left(\mathbf{x}_{m} \mathbf{y}_{k}\right)\right] d S \\
& +\sum_{l=1}^{L} W\left(\mathbf{x}_{m}, \mathbf{y}_{l}, p\right)+\sum_{K}\left[q_{c} w_{c}\right], \quad \forall \mathbf{x} \in \partial \Omega_{n},
\end{aligned}
$$




$$
\begin{aligned}
\frac{1}{2} \theta_{n}\left(\mathbf{x}_{m}\right)=\sum_{k=1}^{N}[ & v_{n}\left(\mathbf{y}_{k}\right) W_{n}\left(\mathbf{x}_{m} \mathbf{y}_{k}\right)+m_{n}\left(\mathbf{y}_{k}\right) \Theta_{n}\left(\mathbf{x}_{m} \mathbf{y}_{k}\right) \\
& \left.-\theta_{n}\left(\mathbf{y}_{k}\right) M_{n}\left(\mathbf{x}_{m} \mathbf{y}_{k}\right)-w\left(\mathbf{y}_{k}\right) V_{n}\left(\mathbf{x}_{m} \mathbf{y}_{k}\right)\right] d S \\
& +\sum_{l=1}^{L} W_{n}\left(\mathbf{x}_{m}, \mathbf{y}_{l}, p\right)+\sum_{K}\left[q_{c} \partial_{n} w_{c}\right], \quad \forall \mathbf{x} \in \partial \Omega_{n},
\end{aligned}
$$

where

$$
\begin{aligned}
W\left(\mathbf{x}_{m}, \mathbf{y}_{k}\right) & =\int_{\partial \Omega_{k}} W\left(\mathbf{y}, \mathbf{x}_{m}\right) d S, \\
\Theta\left(\mathbf{x}_{m}, \mathbf{y}_{k}\right) & =\int_{\partial \Omega_{k}} \Theta\left(\mathbf{y}, \mathbf{x}_{m}\right) d S, \\
\Theta\left(\mathbf{x}_{m}, \mathbf{y}_{k}\right) & =\int_{\partial \Omega_{k}} \Theta\left(\mathbf{y}, \mathbf{x}_{m}\right) d S, \\
\Theta\left(\mathbf{x}_{m}, \mathbf{y}_{k}\right) & =\int_{\partial \Omega_{k}} \Theta\left(\mathbf{y}, \mathbf{x}_{m}\right) d S, \\
W_{n}\left(\mathbf{x}_{m}, \mathbf{y}_{k}\right) & =\int_{\partial \Omega_{k}} W_{n}\left(\mathbf{y}, \mathbf{x}_{m}\right) d S, \\
\Theta_{n}\left(\mathbf{x}_{m}, \mathbf{y}_{k}\right) & =\int_{\partial \Omega_{k}} \Theta\left(\mathbf{y}, \mathbf{x}_{m}\right) d S, \\
M_{n}\left(\mathbf{x}_{m}, \mathbf{y}_{k}\right) & =\int_{\partial \Omega_{k}} M_{n}\left(\mathbf{y}, \mathbf{x}_{m}\right) d S, \\
W_{n}\left(\mathbf{x}_{m}, \mathbf{y}_{l}, p\right) & =\int_{\Omega_{l}} p(\mathbf{y}) W\left(\mathbf{y}, \mathbf{x}_{m}\right) d \Omega . \\
W\left(\mathbf{x}_{m}, \mathbf{y}_{l}, p\right) & =\int_{\Omega_{l}} p(\mathbf{y}) W\left(\mathbf{y}, \mathbf{x}_{m}\right) d \Omega, \\
\left.V_{n}\right) & \int_{\partial \Omega_{k}}\left(\mathbf{y}, \mathbf{x}_{m}\right) d S,
\end{aligned}
$$

System of (42) consist of $2 N+K$ equations, where $2 N$ corresponds to number of BEs and $K$ corresponds to number corner points. For convenience it can be presented in the matrix form

$$
\begin{aligned}
\frac{1}{2}\left|\begin{array}{c}
w \\
\theta_{n}
\end{array}\right|= & \left|\begin{array}{cc}
{[W]} & {[\Theta]} \\
{\left[W_{n}\right]} & {\left[\Theta_{n}\right]}
\end{array}\right| \cdot\left|\begin{array}{c}
{\left[v_{n}\right]} \\
{\left[m_{n}\right]}
\end{array}\right|-\left|\begin{array}{cc}
{[M]} & {[V]} \\
{\left[M_{n}\right]} & {\left[V_{n}\right]}
\end{array}\right| \\
& \cdot\left|\begin{array}{c}
{\left[\theta_{n}\right]} \\
{[w]}
\end{array}\right|+\left|\begin{array}{c}
{[W]} \\
{\left[W_{n}\right]}
\end{array}\right| \cdot\left|\begin{array}{c}
{[p]} \\
{[p]}
\end{array}\right|
\end{aligned}
$$

or

$$
[A] \cdot\{X\}=[P] .
$$

Here $[A]$ is the matrix of the dimension $(2 N+K) \cdot(2 N+K)$ that depends on boundary conditions, elements of which are functions defined by (44), $X$ is column vector of unknown functions of the dimension $2 N+K, P$ and is column vector of known functions of the dimension $2 N+K$ which depend on boundary conditions, external load, and discretization of the domain $\Omega$ and its boundary $\partial \Omega$.

For any specific external load and boundary conditions from system (46) can be constructed system (47). Matrix $A$ and vector $P$ can be calculated using (44).

\section{Calculation Integrals over Boundary and Domain Elements}

There are two approaches to calculation of integrals (44) over the BE. The first one explores numerical calculations using quadrature formulas. The second one consists in analytical integrations of corresponding integrals.

Numerically integrals over boundary elements are usually calculated using the Gaussian quadrature formulas [20, 21]

$$
\int_{a}^{b} p(y) f(y) d x \cong \sum_{q=1}^{Q} \omega_{q} f\left(y_{q}\right)+R(f),
$$

where $Q$ is the number of nodes, $[a, b]$ is any finite or infinite segment of the real line, $y_{q}$ are coordinates of the nodes, $\omega_{q}$ is the weighting factor, $p(y)$ is the weighting function, and $R(f)$ is the remainder of the quadrature. It is necessary that product of functions $f(y)$ and $p(y)$ is integrable on $[a, b]$. The coordinates $y_{q}$ of nodes and the weighting factor $\omega_{q}$ can be found in $[20,21]$.

The Gaussian quadrature formulas can be effectively applied for calculation of the integrals without singularities, in the case of integrals (44) only for $x_{m} \notin \partial \Omega_{k}$. In the case of singular boundary elements, when $x_{m} \in \partial \Omega_{k}$, formulas (48) cannot be applied. In this case special regularization technique has to be applied.

Let us introduce the system of coordinates that related to the $\mathrm{BE}$ with number $m$; axis $x_{1}$ coincides with that $\mathrm{BE}$ and axis $x_{2}$ is perpendicular to it, as it is shown in Figure 6. Calculation of the regular and divergent integral will be done in that system of coordinates.

7.1. Calculation of the Divergent Integrals. In the above mentioned system of coordinates, the local coordinates of the points $\mathbf{x}^{k}$ and $\mathbf{y}^{m}$ have the form

$$
x_{1}^{m}=0, \quad x_{2}^{m}=0, \quad y_{1}^{m}=t, \quad y_{2}^{m}=0,
$$

where $t \in\left[-\Delta_{m}, \Delta_{m}\right]$ is a local coordinate.

Therefore, fundamental solutions on the singular boundary element have the form [17]

$$
W\left(\mathbf{x}_{m}, \mathbf{y}\right)=\frac{1}{8 \pi D} t^{2} \ln |t|
$$

$$
\begin{gathered}
M\left(\mathbf{x}_{m}, \mathbf{y}\right)=-\frac{1}{8 \pi}[2 v+(1+v)(1+2 \ln |t|)], \\
\Theta_{n}\left(\mathbf{x}_{m}, \mathbf{y}\right)=-\frac{1}{8 \pi D}(1+2 \ln |t|), \\
V_{n}\left(\mathbf{x}_{m}, \mathbf{y}\right)=\frac{1+v}{4 \pi t^{2}} \\
\Theta\left(\mathbf{x}_{m}, \mathbf{y}\right)=V\left(\mathbf{x}_{m}, \mathbf{y}\right)=W_{n}\left(\mathbf{x}_{m}, \mathbf{y}\right)=M_{n}\left(\mathbf{x}_{m}, \mathbf{y}\right)=0 .
\end{gathered}
$$


Integrals in (50) are not complicate and can be calculated analytically using regularization technique developed in [16]. As result we obtain

$$
\begin{gathered}
W\left(\mathbf{x}_{m}, \mathbf{y}_{m}\right)=\frac{\Delta_{m}^{3}}{36 \pi D}\left(3 \ln \Delta_{m}-1\right), \\
M\left(\mathbf{x}_{m}, \mathbf{y}_{m}\right)=-\frac{\Delta_{m}}{4 \pi}\left[v-1+2(1+v) \ln \Delta_{m}\right], \\
\Theta_{n}\left(\mathbf{x}_{m}, \mathbf{y}_{m}\right)=-\frac{\Delta_{m}}{4 D}\left(2 \ln \Delta_{m}-1\right), \\
V_{n}\left(\mathbf{x}_{m}, \mathbf{y}_{m}\right)=\frac{1-v}{2 \pi \Delta_{m}} .
\end{gathered}
$$

In (51) it has been taken into account that integral containing kernel $W$ is regular and no special consideration is needed. The integrals containing kernels $M$ and $\Theta_{n}$ are strongly singular; they have to be considered in the sense of Cauchy principle value. The integral containing kernel $V$ is hypersingular; it has to be considered in the sense of Hadamard' finite part. For more information refer to $[13,14,22]$.

7.2. Calculation of the Regular Integrals. In the system of coordinates presented in Figure 6 coordinates of point $\mathbf{x}^{k}$ and $\mathrm{y}$ are

$$
\begin{array}{ll}
x_{1}^{m}=0, & y_{1}=y_{1}^{k}+t \cos \gamma, \\
x_{2}^{m}=0, & y_{2}=y_{2}^{k}-t \sin \gamma,
\end{array}
$$

where $y_{1}^{k}, y_{2}^{k}$ are coordinates of the middle of the BE with number $k, y$ is the angle between vectors $\mathbf{n}_{m}$ and $\mathbf{n}_{k}$ that are perpendicular to the BEs with numbers $m$ and $k$, respectively counted in clockwise direction. Fundamental solutions in this case are regular and are presented by $(28)-(30)$. After analytical integration they can be presented in the following form [17]:

$$
\begin{aligned}
W\left(\mathbf{x}_{m}, \mathbf{y}_{k}\right)= & \frac{1}{8 \pi D}\left(r^{2} I_{1}+2 B I_{2}+I_{3}\right), \\
\Theta\left(\mathbf{x}_{m}, \mathbf{y}_{k}\right)= & \frac{C}{4 \pi D}\left(I_{1}+\Delta_{k}\right), \\
M\left(\mathbf{x}_{m}, \mathbf{y}_{k}\right)= & -\frac{1}{4 \pi}\left((1+\nu)\left(I_{1}+\Delta_{k}\right)+I_{1,0}\left(C^{2}+v B^{2}\right)\right. \\
& \left.+2 I_{1,1} \nu B+I_{1,2} \nu\right), \\
V\left(\mathbf{x}_{m}, \mathbf{y}_{k}\right)= & -\frac{C}{4 \pi}\left[I_{1,0}(5-v)-2 I_{2,0}\left(C^{2}+v B^{2}(2-v)\right)\right. \\
W\left(\mathbf{x}_{m}, \mathbf{y}_{k}\right)= & -\frac{1}{4 \pi D}\left(y_{2}\left(I_{1}+\Delta_{k}\right)-I_{2} \sin \gamma\right), \\
\Theta_{n}\left(\mathbf{x}_{m}, \mathbf{y}_{k}\right)= & -\frac{1}{4 \pi D} \\
& \times\left[-\left(I_{1}+\Delta_{k}\right) \cos \gamma+C\left(I_{1,0} y_{2}-I_{1,1} \sin \gamma\right)\right] ;
\end{aligned}
$$

$$
\begin{aligned}
& M_{n}\left(\mathbf{x}_{m}, \mathbf{y}_{k}\right) \\
& =-\frac{1}{4 \pi}\left[I _ { 1 , 0 } \left(2 B \sin \gamma-2 C \cos \gamma-(1+\nu) y_{2}\right.\right. \\
& \left.+I_{1,1}(1+3 \nu \cos \gamma)\right) \\
& +2 I_{2,0} y_{2}\left(C^{2}+B^{2} v\right)+2 I_{2,0} y_{2}\left(C^{2}+B^{2} v\right) \\
& +2 I_{2,1} \times\left(2 B \nu y_{2}-\left(C^{2}+B^{2} \nu\right) \sin \gamma\right) \\
& \left.+2 I_{2,2} \nu\left(y_{2}-2 B \sin \gamma\right),-2 I_{2,3} \nu \sin \gamma\right], \\
& V_{n}\left(\mathbf{x}_{m}, \mathbf{y}_{k}\right) \\
& =-\frac{1}{4 \pi}\left[I_{1,0}(\nu-5) \cos \gamma+2 I_{2,0}\right. \\
& \left(C(5-v) y_{2}+B(2-v)\right. \\
& \left.\times(B \cos \gamma-2 C \sin \gamma)+3 C^{2} \cos \gamma\right) \\
& +2 I_{2,1}(-C(5-v) \sin \gamma+2(2-\nu) \\
& \times(B \cos \gamma-C \sin \gamma))+2 I_{2,2}(2-\nu) \\
& \times \cos \gamma-8 I_{3,0} C y_{2}\left(C^{2}+(2-\nu) B^{2}\right) \\
& -8 I_{3,1} C\left(-C^{2} \sin \gamma+B(2-\nu)\right. \\
& \left.\times\left(2 y_{2}-B \sin \gamma\right)\right)-8 I_{3,2}(2-\gamma) \\
& \left.\times\left(y_{2}-2 B \sin \gamma\right) \times C+8 I_{3,3} C(2-\nu) \sin \gamma\right] .
\end{aligned}
$$

Here $B=y_{1} \cos \gamma-y_{2} \sin \gamma, D=\operatorname{arctg}\left(\Delta_{k}+B\right) / C-$ $\operatorname{arctg}\left(-\Delta_{k}+B\right) / C, C=y_{1} \sin \gamma+y_{2} \cos \gamma$,

$$
\begin{aligned}
& F=\frac{F_{1}}{F_{2}}=\ln \frac{\left|\Delta_{k}^{2}+2 B \Delta_{k}+r^{2}\right|}{\left|\Delta_{k}^{2}-2 B \Delta_{k}+r^{2}\right|}, \\
& r=|\mathbf{y}-\mathbf{x}|=\left(y_{1}^{2}+y_{2}^{2}\right)^{1 / 2}, \\
& I_{n, m}=\int_{-\Delta_{k}}^{\Delta_{k}} \frac{t^{m} d t}{\left(t^{2}+2 B t+r^{2}\right)^{n}}, \quad n=1,2,3, \quad m=1,2,3, \\
& I_{n}=\int_{-\Delta_{k}}^{\Delta_{k}} t^{n} \ln r d t, \quad n=0,1,2 .
\end{aligned}
$$

7.3. Calculation of the Domain Integrals. In the BIE can appear also domain integrals (25), which are used to take into account action of the load distributed over domain on the plate. They are regular and can be calculated numerically using the Gaussian quadratures $[20,21]$. 


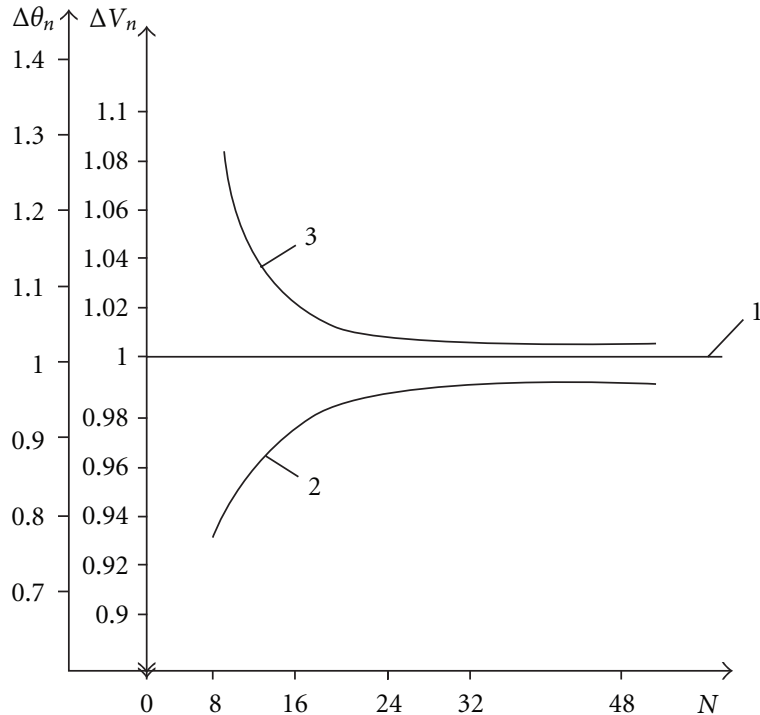

Figure 8: Dependence of the boundary data $\Delta \theta_{n}$ and $\Delta v_{n}$ on number of the BE.

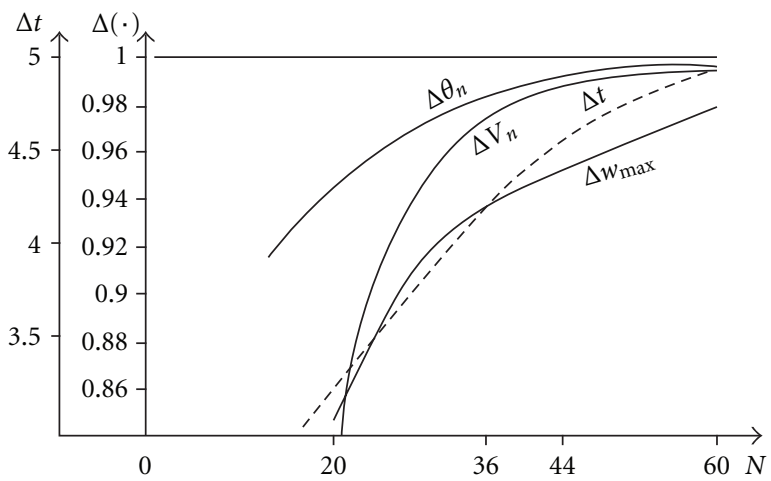

Figure 9: Dependence of the boundary data and time of calculations on number of the $\mathrm{BE}$.

In order to calculate integrals over domain $\Omega$ we divide it into $L$ triangular elements as it is shown in Figure 7. Integrals in (45) over triangle can be calculated in the form

$$
\begin{array}{r}
W\left(\mathbf{x}_{m}, \mathbf{y}_{l}, p\right)=\sum_{q=1}^{Q} p\left(\mathbf{y}_{q}\right) W\left(\mathbf{x}_{m}, \mathbf{y}_{q}\right) \omega_{q}, \\
W_{n}\left(\mathbf{x}_{m}, \mathbf{y}_{l}, p\right)=\sum_{q=1}^{Q} p\left(\mathbf{y}_{q}\right) W_{n}\left(\mathbf{x}_{m}, \mathbf{y}_{q}\right) \omega_{q}, \\
\forall \mathbf{x}_{m} \in \Omega,
\end{array}
$$

where $y_{q}$ are coordinates of nodes on the triangular element, $\omega_{q}$ is the weighting factor. Coordinates $y_{q}$ of nodes and weighting factor $\omega_{q}$ for triangle can be found in $[20,21]$.

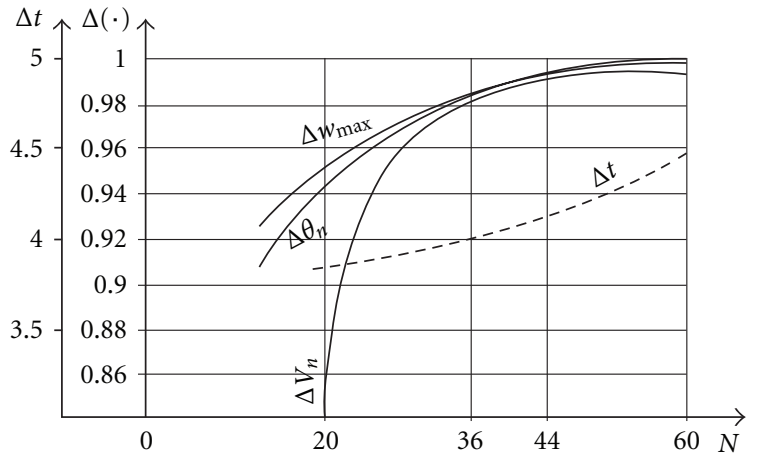

FIGURE 10: Dependence of the boundary data and time of calculations on number of the BE.

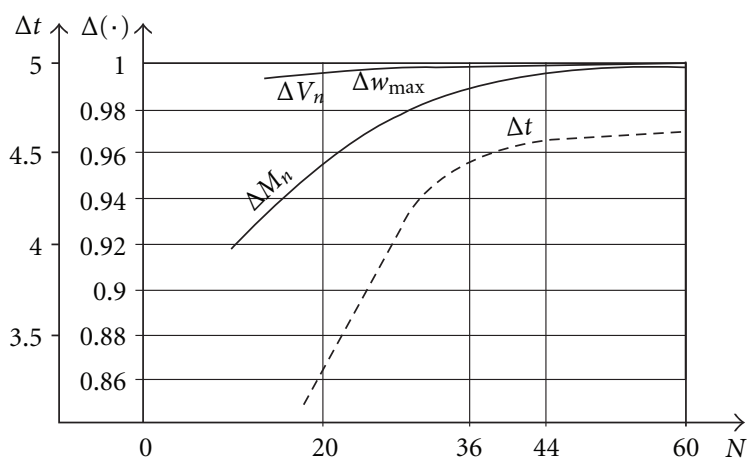

Figure 11: Dependence of the boundary data and time of calculations on number of the BE.

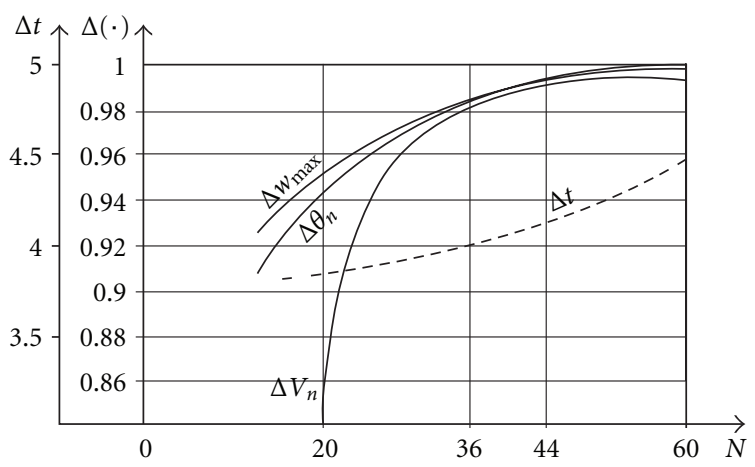

Figure 12: Dependence of the boundary data and time of calculations on number of the BE.

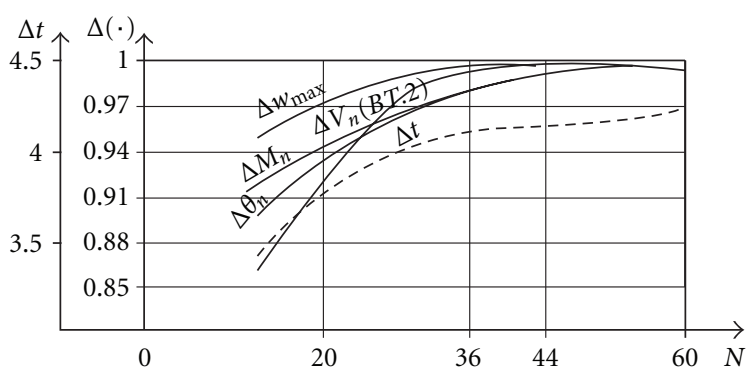

FIgURE 13: Dependence of the boundary data and time of calculations on number of the BE. 
TABle 1

\begin{tabular}{ccccccr} 
Loading pattern & Number of BE & Points & & \multicolumn{2}{c}{ Boundary conditions } & $\Delta t$ \\
& & & $\Delta w$ & $\Delta \theta_{n}$ & $\Delta M_{n}$ & $\Delta V_{n}$ \\
\hline \\
\hline
\end{tabular}

TABle 2

\begin{tabular}{|c|c|c|c|c|c|c|c|c|}
\hline \multirow[t]{2}{*}{ Loading pattern } & \multirow{2}{*}{ Number of BE } & \multirow[t]{2}{*}{ Points } & \multicolumn{4}{|c|}{ Boundary conditions } & \multirow[t]{2}{*}{$\Delta t$} & \multirow[t]{2}{*}{$\Delta w_{\max }$} \\
\hline & & & $\Delta w$ & $\Delta \theta_{n}$ & $\Delta M_{n}$ & $\Delta V_{n}$ & & \\
\hline $\mathrm{i}$ & & & & & & & & \\
\hline$\iota^{1}$ & 20 & 1 & - & 0.940 & - & 0.849 & 3.831 & 0.950 \\
\hline$\frac{i}{1}$ & 36 & 1 & - & 0.985 & - & 0.980 & 3.952 & 0.983 \\
\hline$\underset{\uparrow \uparrow \uparrow \uparrow \uparrow \uparrow \uparrow \uparrow}{\nabla} q$ & & & & & & & & \\
\hline 9 & 44 & 1 & - & 0.993 & - & 0.993 & 4.154 & 0.988 \\
\hline
\end{tabular}

TABle 3

Loading pattern

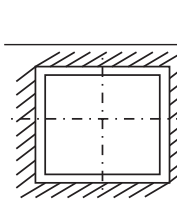

䲝个个遥。

Number of BE Points

Boundary conditions

$\Delta t \quad \Delta w_{\max }$

\begin{tabular}{cccccccc}
\multicolumn{1}{c}{$\Delta w$} & $\Delta \theta_{n}$ & $\Delta M_{n}$ & $\Delta V_{n}$ & & \\
20 & 1 & - & - & 0.956 & 0.997 & 4.251 & 0.995 \\
36 & 1 & - & - & 0.990 & 0.997 & 5.462 & 0.996 \\
44 & 1 & - & - & 0.995 & 0.999 & 5.564 & 0.998
\end{tabular}

TABLE 4

\begin{tabular}{|c|c|c|c|c|c|c|c|c|c|}
\hline \multirow{2}{*}{ Loading pattern } & & \multirow{2}{*}{ Number of BE } & \multirow{2}{*}{ Points } & \multicolumn{4}{|c|}{ Boundary conditions } & \multirow{2}{*}{$\Delta t$} & \multirow[t]{2}{*}{$\Delta w_{\max }$} \\
\hline & & & & $\Delta w$ & $\Delta \theta_{n}$ & $\Delta M_{n}$ & $\Delta V_{n}$ & & \\
\hline \multirow{4}{*}{ 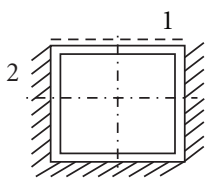 } & \multirow{6}{*}{ 哆 } & \multirow{2}{*}{20} & 1 & - & 0.935 & - & 0.997 & \multirow{2}{*}{2.631} & \multirow{2}{*}{0.969} \\
\hline & & & 2 & - & - & 0.945 & 0.968 & & \\
\hline & & \multirow{2}{*}{36} & 1 & - & 0.985 & - & 0.990 & \multirow{2}{*}{4.529} & \multirow{2}{*}{0.989} \\
\hline & & & 2 & - & 一 & 0.987 & 0.997 & & \\
\hline \multirow{2}{*}{ 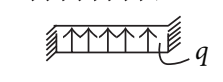 } & & \multirow{2}{*}{44} & 1 & - & 0.993 & - & 0.992 & \multirow{2}{*}{4.761} & \multirow{2}{*}{0.992} \\
\hline & & & 2 & - & - & 0.994 & 1.000 & & \\
\hline
\end{tabular}

TABle 5

\begin{tabular}{|c|c|c|c|c|c|c|c|c|c|}
\hline \multirow{2}{*}{ Loading pattern } & & \multirow{2}{*}{ Number of BE } & \multirow{2}{*}{ Points } & \multicolumn{4}{|c|}{ Boundary conditions } & \multirow{2}{*}{$\Delta t$} & \multirow[t]{2}{*}{$\Delta w_{\max }$} \\
\hline & & & & $\Delta w$ & $\Delta \theta_{n}$ & $\Delta M_{n}$ & $\Delta V_{n}$ & & \\
\hline \multirow{4}{*}{ 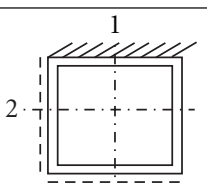 } & \multirow{6}{*}{ 出 } & \multirow{2}{*}{20} & 1 & - & - & 0.945 & 0.967 & \multirow{2}{*}{3.671} & \multirow{2}{*}{0.970} \\
\hline & & & 2 & - & 0.937 & - & 0.926 & & \\
\hline & & \multirow{2}{*}{36} & 1 & - & - & 0.987 & 0.998 & \multirow{2}{*}{4.239} & \multirow{2}{*}{0.999} \\
\hline & & & 2 & - & 0.985 & - & 0.995 & & \\
\hline \multirow{2}{*}{ 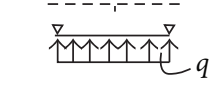 } & & \multirow{2}{*}{44} & 1 & - & - & 0.994 & 0.999 & \multirow{2}{*}{4.281} & \multirow{2}{*}{0.996} \\
\hline & & & 2 & - & 0.993 & - & 0.993 & & \\
\hline
\end{tabular}


TABLE 6

\begin{tabular}{|c|c|c|c|c|c|c|c|c|}
\hline \multirow{2}{*}{ Loading pattern } & \multirow{2}{*}{ Number of BE } & \multirow{2}{*}{ Points } & \multicolumn{4}{|c|}{ Boundary conditions } & \multirow{2}{*}{$\Delta t$} & \multirow[t]{2}{*}{$\Delta w_{\max }$} \\
\hline & & & $\Delta w$ & $\Delta \theta_{n}$ & $\Delta M_{n}$ & $\Delta V_{n}$ & & \\
\hline \multirow{6}{*}{ 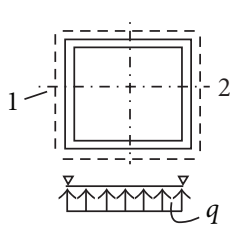 } & \multirow{2}{*}{20} & 1 & 0.947 & 0.995 & - & - & \multirow{2}{*}{4.171} & \multirow{2}{*}{0.936} \\
\hline & & 2 & - & 0.937 & - & 0.817 & & \\
\hline & \multirow{2}{*}{36} & 1 & 0.980 & 0.998 & - & - & \multirow{2}{*}{4.219} & \multirow{2}{*}{0.976} \\
\hline & & 2 & - & 0.984 & - & 0.973 & & \\
\hline & \multirow{2}{*}{44} & 1 & 0.993 & 0.999 & - & - & \multirow{2}{*}{4.461} & \multirow{2}{*}{0.983} \\
\hline & & 2 & - & 0.992 & - & 0.995 & & \\
\hline
\end{tabular}

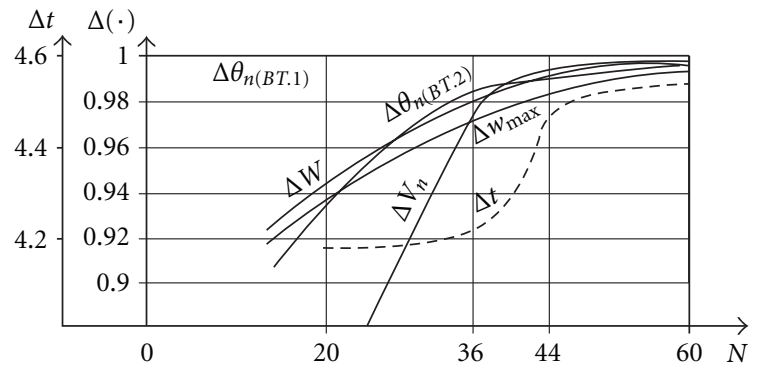

Figure 14: Dependence of the boundary data and time of calculations on number of the BE.

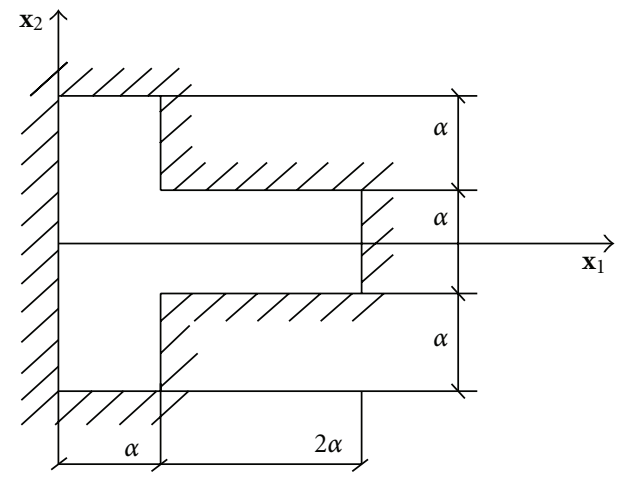

Figure 15: T-shaped plate.

\section{Numerical Examples}

We have considered here some benchmark examples that correspond to bending of the thin plates of different shape. In all examples the ratio $2 h / R=0.1$ has been used.

8.1. Calculation of the Circular Plate. First let us consider circular simply supported over-the-contour plate that subjected to action the uniformly distributed over the domain $\Omega$ load. For this case analytical solution has the form [23]

$$
w(\rho)=\frac{P \rho^{2}}{16 D(1+\nu)}\left(\rho^{2}-R^{2}\right),
$$

where $\rho$ is the radial polar coordinate, $R$ is the radius of the plate.
Expressions for $\theta_{n}, m_{n}, v_{n}$ can be obtained from (57) applying differential operators (3). Dependence of the accuracy of the BEM on number $N$ of the BE has been studied here. In all occurrences for approximation of the domain $\Omega$ it was divided by 288 triangular elements. Results of calculations of the unknown boundary data are presented on Figure 8, where curve 1 corresponds to analytical solution, curve 2 corresponds to $\Delta \theta_{n}=\theta_{n}^{\text {appr }} / \theta_{n}^{\text {extact }}$, and curve 3 corresponds to $\Delta v_{n}=v_{n}^{\text {appr }} / v_{n}^{\text {extact }}$, respectively.

Analysis of the data presented in Figure 8 shows that generalized shear force $v_{n}$ is calculated more accurately. For example, calculation accuracy to within $5 \%$ for $v_{n}$ is reached for $N=13$, whereas the same accuracy for the slope of the element perpendicular to the middle surface $\theta_{\mathrm{n}}$ is reached for $N=24$.

In order to compare traditional and proposed here analytical approach, evaluation of integrals (44) using the Gaussian quadratures (48) and presented here equations (52) has been done for circular simply supported over-thecontour plate. In both cases divergent integrals have been calculated using (50) and $N=32$ rectilinear BE. Time for calculation of the above-mentioned integrals in traditional approach has to be four times longer in order to reach the same accuracy as in proposed here analytical approach.

8.2. Calculation of the Rectangular Plate. We consider here some benchmark examples for rectangular plate with different boundary conditions. In the first example plate was loaded by concentrated force applied to the point in its center. In all other examples the plate was loaded by uniformly distributed over the domain $\Omega$ load. Boundary conditions and load are presented in the first column of Tables 1-6. For all presented here examples solution obtained by BEM was compared with existing analytical solution, which can be presented in the form of Fourier series [23].

Columns 4-7 of Tables 1-6 show $\Delta w, \Delta \theta_{n}, \Delta m_{n}, \Delta v_{n}-$ the ratio of the unknown boundary conditions for different values of $N$ to the values obtained analytically at points 1 and 2 for the schemes presented in the column 1 . This data can be used to optimize the number of the BE depending on the required accuracy for different boundary conditions. According to our estimates, the value $N=40$ can be recommended in calculations performed for different combinations of boundary conditions and loading schemes. Here, the error in the calculation of the unknown boundary conditions does not exceed 1\%. Column 9 of Tables 1-6 

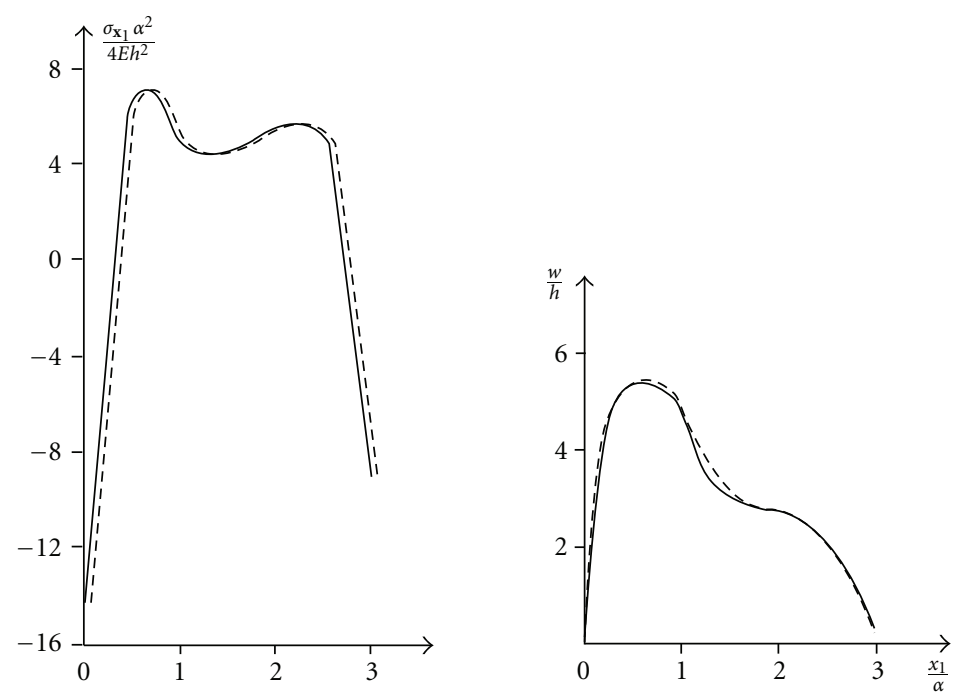

Figure 16

shows values of $\Delta w_{\max }$ - ratio of the maximum deflection calculated by the BEM to the corresponding values obtained analytically [23]. With number of the BE equal to $N=40$, the error is no greater than $1.7 \%$ for any variant of boundary conditions and loading presented in Tables 1-6 except the first one. It is equal to $4.5 \%$ for the first case, which can be attributed to the inaccuracy of approximation of the concentrated force.

Integrals in (44) have been calculated numerically using the Gaussian quadratures (48) and analytically using presented here equations (52). An important fact testifying to the expediency of use of the presented here equations for analytical integration (52) for calculation of the coefficients of system (47) is the reduction of the computational time. Column 8 of the Tables 1-6 shows values of $\Delta t$ - the ratio of the time required to construct the system of the linear algebraic equations (47) analytically using (52) and numerically using the Gaussian quadratures. Studies have shown that use of the approach proposed here makes it possible to cut the time required to obtain solution the problem by the factor from 2.5 to 5 . This is particularly important when the calculations have to be performed repeatedly, such as in the solution of the dynamic and nonlinear problems. The amount of time saved depends mainly on the number of the $\mathrm{BE}$ approximating the boundary, as well as on the type of boundary conditions.

In order to visualize results from Tables 1-6 also Figures 9-14 are presented. They complement data from the corresponding tables and present them in more convenient and visual form.

8.3. Calculation of the T-Shaped Plate. The diagram of the Tshaped plate together with boundary conditions is presented in Figure 15. The plate is subjected to action of the uniformly distributed over the domain $\Omega$ load of the magnitude $q$. Material properties of the plate have been calculated from the equation $\left(q a^{4}\right) /\left(E h^{4}\right)=100$ and Poisson ratio $v=0.3$.
In Figure 16 are presented distributions of the values $\sigma_{x} a^{2} / 4 E h^{2}$ and $w / h$ for the cross-section with coordinate $x_{2}=0$ and for number of the Bes $N=96$. The solid line corresponds to the BEM and dash line to FEM solutions, respectively.

From this data follows that results obtained by the BEM and by the FEM are in a good agreement but time of calculation by the BEM is significantly less.

\section{Conclusion}

Direct BIEM based on Betty's theorem is applied here for solution of thin elastic plate bending problems for different boundary conditions and load. Analytical integration of the regular and divergent integrals over the $\mathrm{BE}$ is applied, and effective formulas for calculation of coefficients of the system of linear algebraic equations for the BEM have been developed. The main advantage of the proposed approach consists in significant reduction of the calculation time comparison with traditional approach based on Gaussian's quadratures. Numerical examples demonstrate effectiveness of the proposed here approach. In all presented examples it was demonstrated high accuracy, in good agreement with existing analytical solutions and significant reduction of the time of calculations in comparison with traditional approaches.

\section{Acknowledgment}

The author is very grateful to his former Ph.D. student Dr. Alexander Lukin from Kharkov State University for help in this paper preparation.

\section{References}

[1] M. H. Aliabadi, "Applications in solids and structures," in The Boundary Element Method, vol. 2, John Wiley \&Sons, 2002. 
[2] F. Hartmann, Introduction to Boundary Elements. Theory and Applications, Springer, 1989.

[3] D. E. Beskos, Ed., Boundary Element Analysis of Plates and Shells, Springer, Berlin, Germany, 1991.

[4] N. J. Altiero and D. L. Sikarskie, "A boundary integral method applied to plates of arbitrary plan form," Computers and Structures, vol. 9, pp. 163-168, 1978.

[5] A. Frangi and M. Guiggiani, "Boundary element analysis of Kirchhoff plates with direct evaluation of hypersingular integrals," International Journal for Numerical Methods in Engineering, vol. 46, pp. 1845-1863, 1999.

[6] A. Frangi and M. Guiggiani, "A direct approach for boundary integral equations with high-order singularities," International Journal for Numerical Methods in Engineering, vol. 49, pp. 871$898,2000$.

[7] F. Hartmann and R. Zormantel, "The direct boundary element method in plate bending," International Journal for Numerical Methods in Engineering, vol. 23, pp. 2049-2069, 1986.

[8] B. Knopke, "The hypersingular integral equation for the bending moments $m_{x x}, m_{x y}$, and $m_{y y}$ of the Kirchhoff plate," Computational Mechanics, vol. 15, pp. 19-30, 1994.

[9] M. Stern, "A general boundary integral formulation for plate bending problems," International Journal for Solids and Structures, vol. 15, pp. 769-782, 1979.

[10] P. H. Wen, M. H. Aliabadi, and A. Young, "Application of dual reciprocity method to plates and shells, engineering analysis with boundary element," International Journal for Numerical Methods in Engineering, vol. 24, pp. 583-590, 2000.

[11] V. V. Zozulya and A. N. Lukin, "Calculation of plates by the method of boundary integral equations under the dynamic loading," Doclady Akademii Nauk of Ukraine, vol. 8, pp. 6470, 1997.

[12] V. V. Zozulya and A. N. Lukin, "Calculation of plates by the boundary integral equations method," Doclady Akademii Nauk of Ukraine, vol. 4, pp. 39-45, 1996.

[13] J. Hadamard, Lectures on Cauchy's Problem in Linear Partial Differential Equations, Yale University Press, New Haven, Conn, USA, 1923.

[14] I. K. Lifanov et al., Hypersingular Integral Equations and Their Applications, Chapman\&Hall, 2004.

[15] V. V. Zozulya, "Regularization of the divergent integrals. I. General consideration," Electronic Journal of Boundary Elements, vol. 4, no. 2, pp. 49-57, 2006.

[16] V. V. Zozulya, "The Regularization of the Divergent Integrals in 2-D Elastostatics," Electronic Journal of Boundary Elements, vol. 7, no. 2, pp. 50-88, 2009.

[17] V. V. Zozulya and A. N. Lukin, "Calculation of plates by the boundary element method," Doclady Akademii Nauk of Ukraine, vol. 4, pp. 60-67, 1996.

[18] M. A. Krasnosel'skii et al., Integral Operators in Spaces of Summable Functions, Noordhoff, 1976.

[19] A. N. Tikhonov and V. Y. Arsenin, Solutions of Ill-Posed Problems, Winston, 1977.

[20] P. Kythe and M. Schaferkotter, Handbook of Computational Methods for Integration, CRC, 2005.

[21] V. Krylov, Approximate Calculation of Integrals, Dover, 2006.

[22] V. V. Zozulya and A. N. Lukin, "Design of plates by the boundary element method," International Applied Mechanics, vol. 33, pp. 241-244, 1997.

[23] S. Timoshenko and S. Woinowski-Krieger, Theory of Plates and Shells, McGraw-Hill, New York, 1959. 

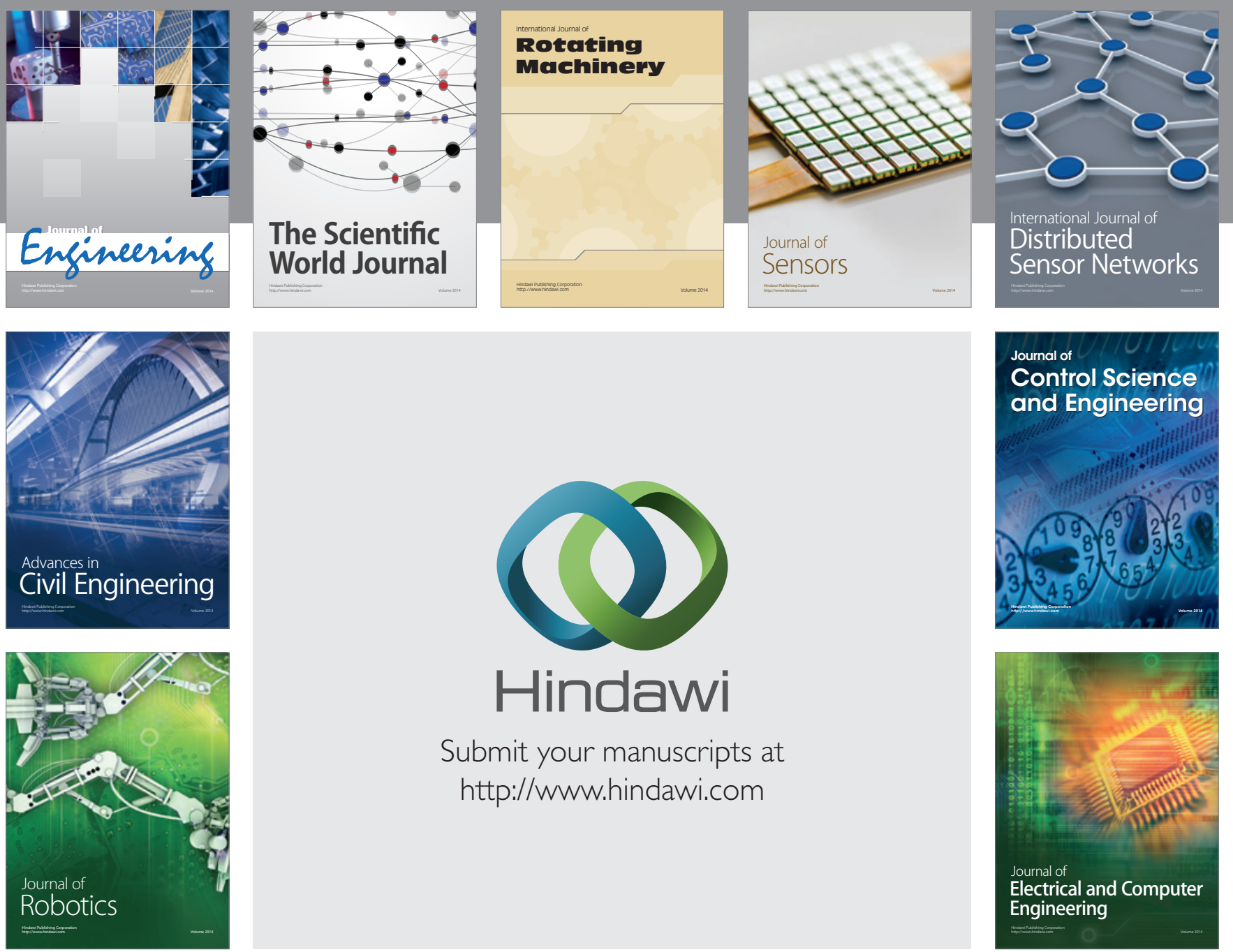

Submit your manuscripts at

http://www.hindawi.com
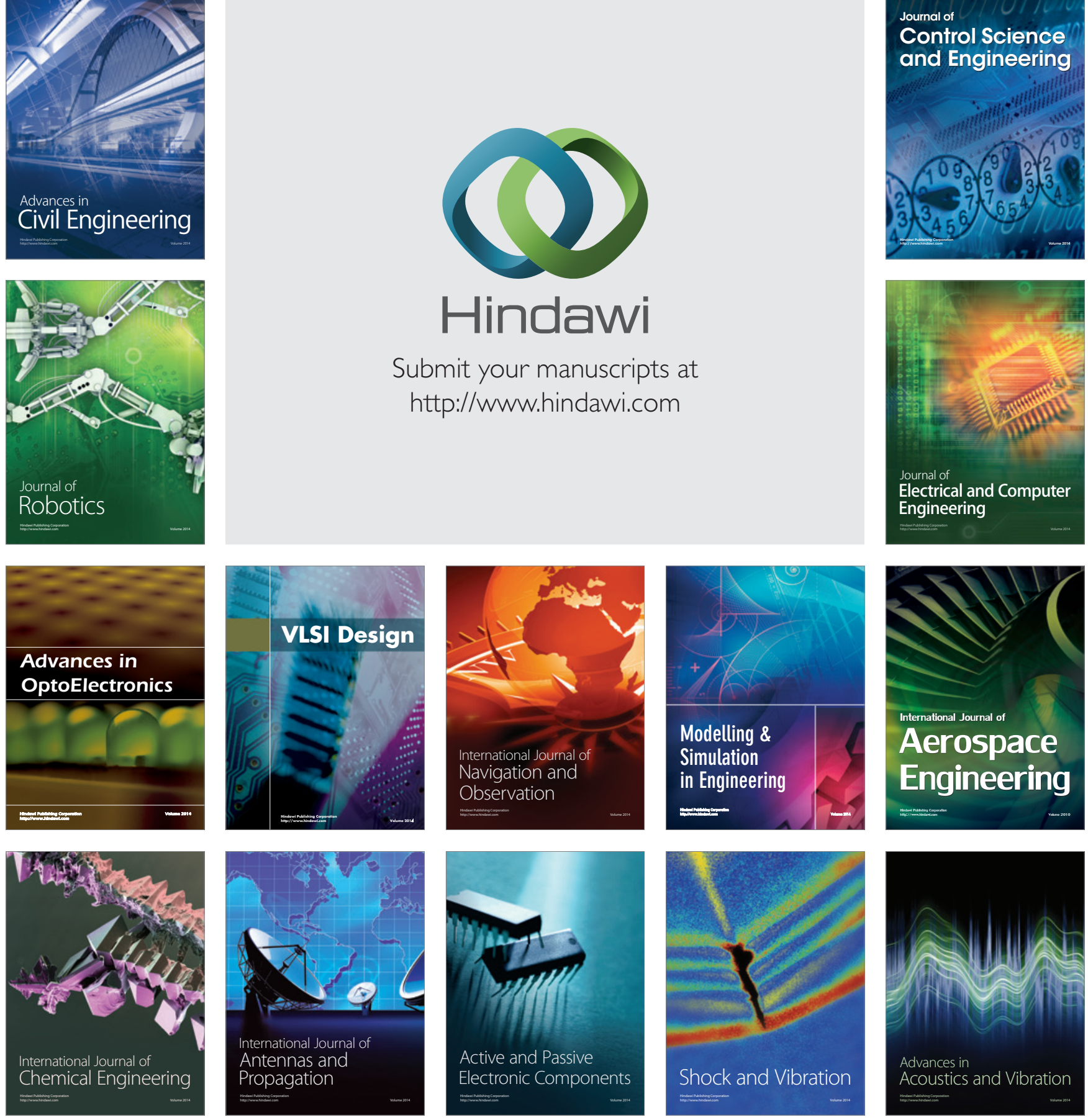